\title{
Técnicas de codificação de canal e modulação para a próxima geração de televisão digital
}

\author{
Leonardo F. Leone, Universidade Presbiteriana Mackenzie, Cristiano Akamine, Universidade \\ Presbiteriana Mackenzie
}

\begin{abstract}
This paper aims to explore the latest and well succeeded techniques for transmission and reception for the next generation of digital terrestrial television broadcasting system, more precisely in study fields of error correction codes and modulation, such as BICM (Bit-Interleaver Coding and Modulation) and NUC (Non Uniform Constellation). There is also an introduction about FoBTV (Future of Broadcast Television), an association created to make the next TV generation unified around the world. An overview of the latest standards generations being developed that employ these techniques is also made.
\end{abstract}

Index Terms-BICM, FoBTV, NUC.

\section{INTRODUÇÃO}

Desde a definição dos primeiros padrões de Televisão digital houve uma grande disputa de mercado entre os padrões desenvolvidos. Essa disputa não foi benéfica em alguns aspectos. A começar pelo consumidor que, por exemplo, ao comprar um dispositivo compatível com um padrão não pode utilizá-lo caso viaje para outro país que não adotou o mesmo padrão. A indústria teve que produzir equipamentos diferentes para locais diferentes do mundo, diminuindo sua escala de produção e aumentando o seu custo. Como curso natural do mercado, as tecnologias referentes à captação, reprodução e armazenamento de conteúdo audiovisual avançaram e houve uma mudança no comportamento do telespectador, que passou a requisitar uma quantidade maior de informação do conteúdo que assiste. $\mathrm{O}$ estilo de consumo de mídia está mudando drasticamente com o desejo de serviços time-shifting, placeshifting, conectados e de múltiplas telas [1].

Partindo da perspectiva exposta, foi criada em 2011 a associação do FoBTV (Future of Broadcast Television). Ela é formada por radiodifusores, fabricantes, operadoras de telecomunicações, organismos de normalização e institutos de pesquisa [2]. Entre os participantes estão entidades como NAB (National Association of Broadcasters), SET (Sociedade Brasileira de Engenharia de Televisão), IEEE Broadcast Technology Society, NHK Science \& Technology Research Laboratories, Video Broadcast Project e ATSC (Advanced

Leonardo F. Leone é Engenheiro de Telecomunicações e mestrando vinculado ao Programa de Pós-Graduação em Engenharia Elétrica e Computação da Universidade Presbiteriana Mackenzie, São Paulo, Brasil (email: lf.leone@yahoo.com.br).

Cristiano Akamine é Professor do Programa de Pós-Graduação em Engenharia Elétrica e Computação da Universidade Presbiteriana Mackenzie, São Paulo, Brasil (e-mail: akamine@ieee.org).
Television Systems Committee). Seu objetivo é desenvolver tecnologias para a próxima geração e fazer recomendações aos organismos de normalização [2]. Um Memorando de Entendimento foi assinado pelos membros. Nele foram abordados assuntos como: objetivos, estrutura da organização, obrigação dos participantes e propriedade intelectual. Do ponto de vista de possíveis cenários de uso da tecnologia, fica clara a necessidade de que os serviços broadcast e broadband sejam integrados. O maior grau de interoperabilidade cai sobre o compartilhamento entre as diversas redes de transmissão [3]. A estrutura da organização ficou definida da seguinte forma: Comitê de Gerenciamento, Secretariado e Comitê Técnico. Sendo o último subdividido nos seguintes grupos: Camada Física, Camada de Rede, Camada de Dados, Gerenciamento e Camada de Aplicação.

Existem inúmeras técnicas sendo estudadas e submetidas a simulações e testes de campo. Algumas ainda em fase de estudo e outras em estágio avançado de operação.

Esse artigo tem o objetivo de descrever as principais pesquisas e avanços tecnológicos para a radiodifusão terrestre. Iniciando pela Seção II, os principais padrões em desenvolvimento são apresentados. Nas Seções III e IV são detalhadas, respectivamente, as técnicas de codificação de canal e modulação adotadas pelos padrões apresentados na Seção II. Por fim, na Seção V, são feitas as considerações finais desse artigo.

\section{PRÓXIMAS GERAÇÕES}

Nesta seção se encontram as atualizações dos principais sistemas de TV digital terrestre, além de novas propostas e desafios.

\section{A. Cloud Transmission}

Cloud Transmission, ou Cloud $T_{X N}$, é uma técnica de transmissão que tem como objetivo o uso eficiente do espectro. Ela permite que mais de um sinal possa trafegar sobre o mesmo espectro. Isso é possível graças à alta robustez do sistema que, segundo [4], deve ter um limiar de SNR de -2 a $-3 \mathrm{~dB}$. O mesmo autor explica que o valor negativo significa que o sistema deve resistir a potências de ruído, co-canal e distorções causadas por multirpercurso superiores ao do sinal desejado. O diagrama da Fig. 1 demonstra como é possível transmitir esses sinais sobre o mesmo espectro. 


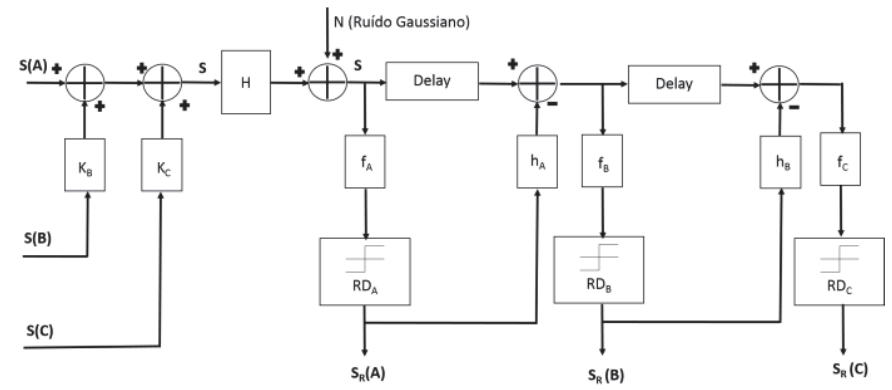

Fig. 1 Diagrama de transmissão e recepção do Cloud Transmission. Adaptado de [5].

Na Fig. 1 é possível observar que $\mathrm{S}(\mathrm{A}), \mathrm{S}(\mathrm{B})$ e $\mathrm{S}(\mathrm{C})$ são os sinais de entrada do sistema e que os sinais $\mathrm{S}(\mathrm{B})$ e $\mathrm{S}(\mathrm{C})$ sofrem atenuação nos blocos $\mathrm{K}_{\mathrm{B}}$ e $\mathrm{K}_{\mathrm{C}}$, respectivamente. A diferenciação entre as potências é necessária para que seja possível a distinção dos sinais no estágio de recepção. Essa diferenciação é chamada de reutilização hierárquica do espectro ou técnica spectrum underlay [5]. Um outro nome dado à mesma técnica é Layered Division Multiplexing (LDM), na qual cada layer, ou camada, é representado por uma stream.

$\mathrm{Na}$ recepção a stream A é decodificada primeiro e retroalimentada para cancelamento no sinal $\mathrm{S}$ recebido. A stream B pode ser decodificada e retroalimentada da mesma maneira, antes da decodificação da stream C [5]. Como a stream A é decodificada primeiro para que as outras sejam decodificadas, existe a obrigatoriedade de que ela seja o sinal mais robusto.

\section{B. ATSC 3.0}

Em março de 2013 o ATSC publicou uma Chamada para Propostas para o ATSC 3.0, na qual são abordados assuntos como requisitos de funcionalidade, modelos de canal utilizados para comparação e técnicas de medida de desempenho. Como pontos em destaque dos requisitos de funcionalidade estão: eficiência espectral, maior taxa e suportar SFN [6]. Em outubro do mesmo ano, o ATSC publicou um sumário das respostas para a Chamada de Propostas presente em [7]. Nele estão reunidas as principais ideias sugeridas por empresas e institutos de pesquisa.

Em abril de 2015 o ATSC demonstrou na NAB Show os elementos-chave do padrão ATSC 3.0 [8]. Dentre os inclusos na definição do padrão estão BICM, LDM, MMT (MPEG media transport), HEVC (High Efficiency Video Coding) e HTML5.

Tendo como foco o ATSC 3.0, em [9] existe uma análise de complexidade do LDM considerando sua implementação em Hardware. Nesse trabalho, foram utilizados no sistema BICM, constelações não uniformes e OFDM. Segundo [9], O OFDM ainda possui parâmetros sob discussão, mas são muito similares àqueles do DVB-T2. O trabalho constata que um sistema com dois layers leva a um aumento de menos de $10 \%$ em uso de memória e lógica do Hardware se comparado com um sistema de um layer.

\section{C. $D V B-T 2$}

O DVB-T2 possui modificações em relação ao DVB-T que resultam em um sistema com grande grau de liberdade de configurações, tornando mais flexível o dimensionamento do mesmo e mais adequado para cada cenário de transmissão.

Pode-se destacar a utilização de PLP's (Physical Layer Pipes), permitindo a transmissão de vários serviços, ou seja, cada PLP corresponde a um sinal de vídeo comprimido que pode ser tratado com parâmetros de codificação e modulação distinto.

Outra novidade trazida pelo DVB-T2 é a configuração das portadoras no símbolo OFDM, na qual existe um modo estendido para $8 \mathrm{~K}, 16 \mathrm{~K}$ e $32 \mathrm{~K}$ de FFT (Fast Fourier Transform). Nesse modo, é possível inserir mais portadoras no mesmo símbolo OFDM do que no modo normal, aumentando assim a eficiência espectral do sistema.

O número de pilotos espalhados inseridos também é um parâmetro configurável no DVB-T2. Oito padrões de piloto que se diferenciam ente si pela quantidade desses dados de referência em um quadro OFDM permitem que a robustez na transmissão do sinal seja condicionada às condições do ambiente em que ele será recebido.

\section{ISDB-Tn}

A próxima geração do ISDB-T prevê a utilização do sistema de transmissão por radiodifusão em conjunto com a Internet. Com esse propósito, a NHK tem desenvolvido uma plataforma para serviços avançados de radiodifusão que integra a tecnologia broadband com broadcast [10]. Com esse intuito, S. Takeuchi, et al relata em [10] que usa HTML5 como método codificação de dados para facilitar a integração dentro de serviços de internet. Além disso, está sendo empregado o esquema de transporte por MMT e que, segundo [10], facilita a entrega híbrida utilizando redes de broadcasting e broadband.

Para a transmissão terrestre de alta capacidade, tem-se estudado o uso de transmissão por dupla polarização (vertical e horizontal) e altas ordens de constelação, como mostrado em [11]. Em [12], existe um relato de teste de campo no qual foi possível verificar a recepção bem-sucedida de uma transmissão em $8 \mathrm{~K}$ a uma distância de $27 \mathrm{Km}$ entre os dois pontos. Isso foi possível fazendo uso de 4096 QAM, dupla polarização com configuração 2x2 MIMO (Multiple In Multiple Out) de antenas e concatenação dos codificadores $\mathrm{BCH}$ e LDPC. A relevância desse trabalho cai não só sobre o fato de se ter atingido uma taxa de 91,8 Mbps com um canal de 6,0 MHz mas também pelo ineditismo, pois segundo [12] este experimento representa a primeira transmissão terrestre em resolução $8 \mathrm{~K}$.

\section{CODIFICAÇÃO DE CANAL}

As técnicas de codificação de canal por FEC (forwarderror-correction) têm sido amplamente estudadas com o intuito de diminuir a probabilidade de decodificação incorreta. $\mathrm{Na}$ prática, isso equivale a permitir que o sistema de recepção opere com valores de SNR (Signal-to-Noise Ratio) cada vez mais baixos.

\section{A. BICM}

O BICM, do inglês Bit-Interleaver Coding and Modulation, é definido como uma cadeia de processos que tratam o fluxo de bits oriundo da codificação de fonte. O primeiro processo 
dessa cadeia é a codificação por BCH. Em seguida o fluxo de bits sofre mais uma codificação através do LDPC. Em uma analogia com o sistema de codificação do ISDB-T, o BCH tem a função de codificação externa enquanto que o LDPC exerce a função de codificação interna. Passados os processos de codificação, o entrelaçador de bits age com o intuito de prevenir possíveis erros concentrados. O último processo dessa cadeia, Modulação, será explorado na Seção IV. O DVB-T2 utiliza tal cadeia para sua transmissão e, segundo [13] o ganho em BER (Bit Error Rate) alcançado após o codificador interno (LDPC versus Codificador Convolucional) é da ordem de $5 \mathrm{~dB}$.

\section{B. $R S-2 D$}

Como novidade na correção de erros, Cloud $T_{X N}$ utiliza uma codificação bidimensional (Product Code) de Reed-Solomon (RS) e LDPC. Ela é formada por uma matriz que pode ser dividida em quatro regiões distintas: dados, paridade do LDPC, paridade do RS e paridade na paridade como mostra a Fig. 2. Nessa estrutura, pode-se considerar o LDPC como codificador interno e o RS como externo.

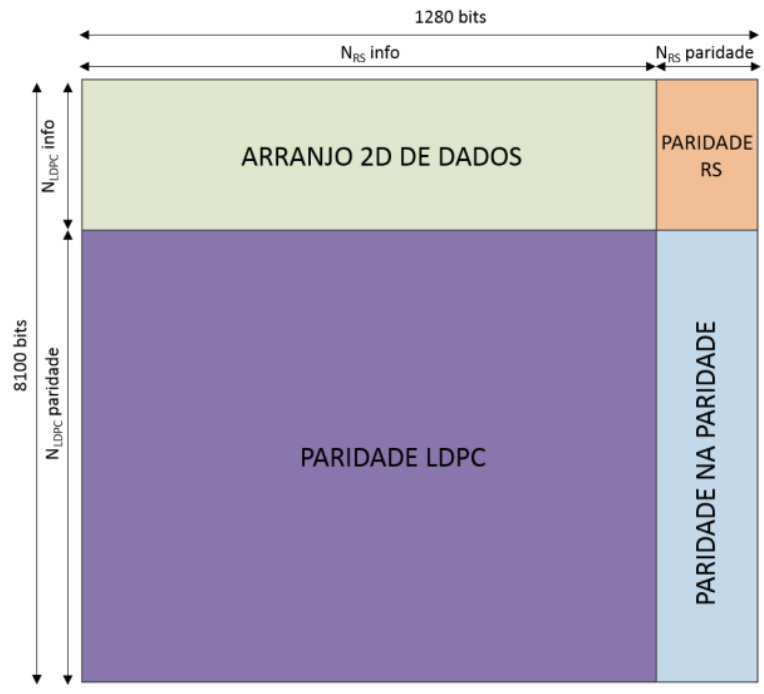

Fig. 2: Product code utilizado no Cloud $T_{X N}$. Adaptado de [14].

J. Montalban, et al, em [14], explicam que o LDPC tem melhor desempenho com curtos erros aleatório, enquanto que o código RS oferece robustez contra erros de rajada e remove o error floor do LDPC.

Outra caraterística a se destacar do RS-2D é o entrelaçamento de bits. O RS-2D é a versão entrelaçada de um código concatenado serialmente [15]. A estrutura de codificação mostrada na Fig. 2 dispensa o entrelaçamento após a codificação, pois o processo de escrita e leitura da matriz proporciona tal entrelaçamento.

De acordo com [15], como o RS, o LDPC e o product code possuem diferentes limiares de decodificação pode-se aplicar diferentes esquemas de decodificação considerando o SNR do sinal recebido. Isso significa dizer que a decodificação pode ser adaptativa, ponderando recursos de processamento a partir das condições de sinal recebido.

Em [14], pode-se destacar as simulações com modelamento de canal AWGN e SFN (Single Frequency Network) com condição de $0 \mathrm{~dB}$ de eco. Ambas com modulação QPSK, taxa de codificação $\mathrm{R}=0,30$, as simulações resultaram em SNR de 1,3dB (AWGN) e 2,1 dB (0 dB de eco) além do limite de Shannon considerando uma capacidade de canal $\mathrm{C}=0,60 \mathrm{bps} / \mathrm{Hz}$.

Em [16] são apresentados resultados de teste de campo para recepção móvel, estipulando diferentes valores de SNR para diferentes cenários de recepção, analisando a viabilidade de um sistema que emprega Cloud $T_{X N}$, bem como estipulando valores de potência de transmissão como referência para planejamento de futuras redes.

\section{MODULAÇÃO}

Afim de se aproximar do limite teórico de capacidade de canal ou aumentar a capacidade de transmissão, estudos têm contemplado o desenvolvimento de novas maneiras de modulação. As modulações por chaveamento de fase (PSK) e por amplitude em quadratura (QAM) continuam sendo as técnicas utilizadas, entretanto as formas e ordens das constelações são alteradas.

\section{A. Constelações não uniformes}

Através de uma distribuição de símbolos diferente da constelação tradicional é possível alterar a capacidade do sistema. Para um esquema BICM, [20] relata que o gap entre o limite de Shannon e a capacidade real do sistema é imposto pelo formato retangular e os níveis igualmente espaçados do QAM uniforme. Segundo [19], técnicas conhecidas como constellation shaping são benéficas para reduzir esse gap, resultando em uma constelação com probabilidade de símbolos desigual.

Os resultados mostrados em [20] são de 256-NUQAM (non uniform QAM) unidimensional e bidimensional. A Fig. 3 mostra ambas as constelações.

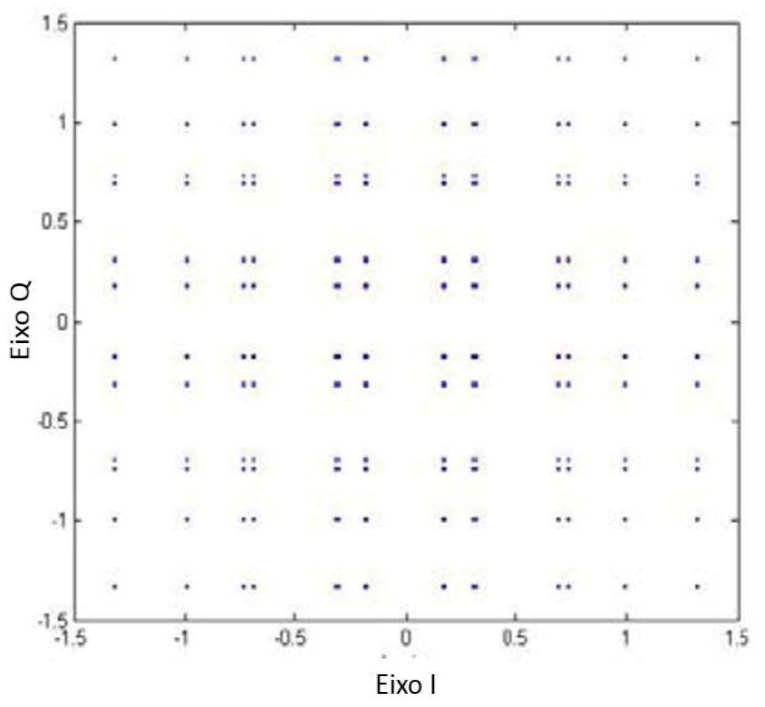

(a) 


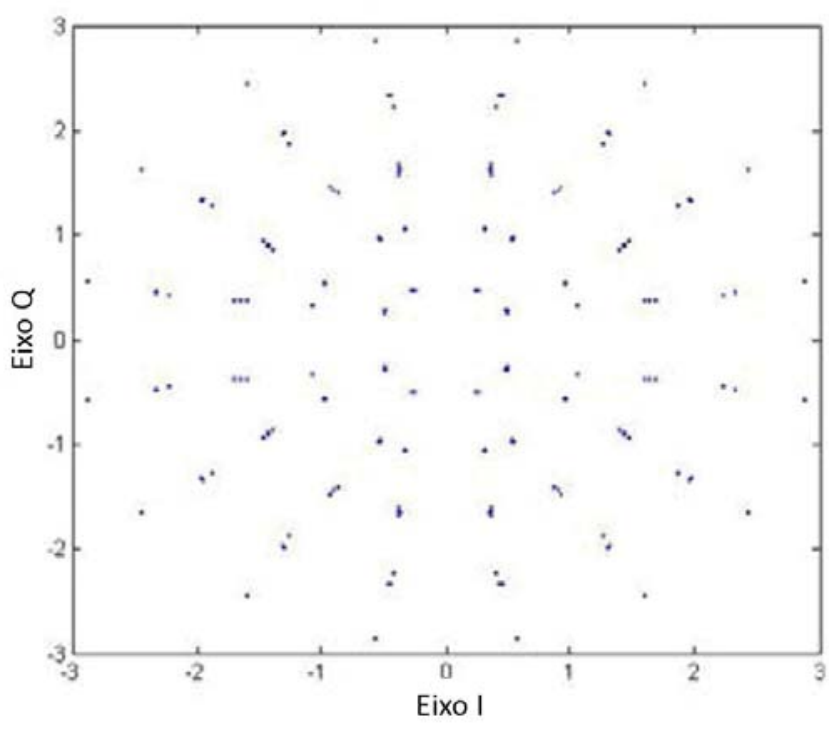

(b)

Fig. 3: Constelações Não Uniformes: 256NUQAM. Adaptado de [20].

(a) 1D-256NUQAM, (b) 2D-

$\mathrm{O}$ unidimensional mantém a estrutura retangular e altera o espaçamento entre símbolos enquanto que o bidimensional altera ambos os parâmetros. Para 1D-1024 NUQAM o ganho observado foi de 1,45 dB, enquanto que para 2D-NUQAM o ganho atingiu $1,55 \mathrm{~dB}$ em relação ao QAM uniforme. Ambos considerando um canal AWGN.

Pode-se também destacar os trabalhos de [17] e [18]. No primeiro, são realizadas simulações com um arranjo circular não uniforme de constelação nos canais AWGN e com multipercurso. O resultado obtido foi que NUC apresenta ganhos para ambos os canais estudados. Em [18], são apresentados resultados de simulação para constelação APSK (Amplitude Phase Shift Keying) com mapeamento gray. Nesse trabalho são propostos dois métodos. No primeiro é alterado o espaçamento dos símbolos em um mesmo anel. O segundo altera a amplitude para cada anel. As simulações contemplaram o modelamento de canal AWGN e os resultados mostraram que para a cadeia BICM ambos os métodos produziram ganho em relação APSK Original.

\section{B. Constelação rotacionada}

O sistema DVB-T2 possui inovações na modulação em relação ao primeiro padrão DVB-T. Além de permitir constelação de maior ordem (até 256-QAM), neste padrão também pode ser habilitada a rotação de constelação. Devido à diferença de valor entre os eixos cartesianos para o mesmo ponto da constelação e ao delay na componente Q introduzido na transmissão, a recepção obtém uma informação redundante de cada ponto da constelação. A importância do delay incide sobre a diversidade. As componentes de um mesmo símbolo são transmitidas em subportadoras distintas dando ao sinal transmitido diversidade em frequência. Mesmo havendo desvanecimento severo em uma das componentes (I ou Q) o ponto é reconstituído através da outra componente. A Fig. 4 demonstra a constelação de um sinal 16-QAM que passou pelo processo explicado.

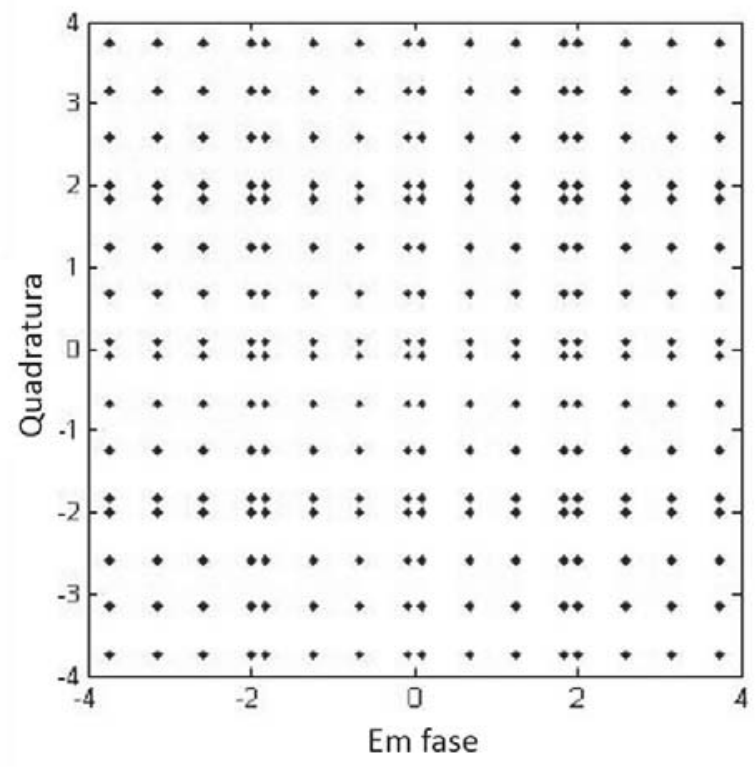

Fig. 4:16-QAM com constelação rotacionada após delay cíclico de Q. Adaptado de [21].

O ganho trazido pela rotação é condicionado ao canal em que o sinal está trafegando e a ordem da constelação. Os resultados obtidos em [21] mostram que os desempenhos dos sistemas com e sem rotação são quase iguais para um canal AWGN e, segundo [22], quanto menor for a ordem da constelação maior o ganho obtido. Isso mostra que tal técnica de modulação é mais vantajosa em sistema de recepção móvel, no qual são utilizadas menores ordens de constelação e a robustez tem maior prioridade que a taxa de bits trafegada.

\section{CONCLUSÃO}

Após a digitalização do sistema de TV existe um novo contexto em que a preocupação se volta ao desenvolvimento de técnicas que garantam à radiodifusão, junto com as telecomunicações, ofertar serviços integrados em redes de alta capacidade de transmissão

Nesse contexto os métodos de correção de erros e modulação são relevantes dentro da camada física do sistema. O desenvolvimento de novas técnicas e aprimoramento das existentes, como mostrado nesse artigo, têm viabilizado a definição dessa camada para a próxima geração de TV digital.

Um sistema implementado com as técnicas apresentadas poderia ser constituído de codificação de vídeo HEVC, esquema de transporte MMT, codificação de canal RS-2D e transmissão LDM. Para a recepção móvel de conteúdo HD poderia ser adotada rotação de constelação. Para a recepção fixa, constelações não uniformes de até 4096 QAM permitiriam transmissão de conteúdo $8 \mathrm{~K}$ a uma taxa de aproximadamente $90 \mathrm{Mbps}$.

\section{REFERÊNCIAS}

[1] FOBTV, "Future of broadcast terrestrial television initiative: memorandum of understanding," Abr. 2012.

[2] FOBTV. About Us. [Online]. Disponível em: http://www.nercdtv.org/fobtv2012/en/aboutus.html 
[3] W. Zhang et al, "Fobtv: Worldwide efforts in developing nextgeneration broadcasting system" IEEE International Symposium on Broadband Multimedia Systems and Broadcasting, vol. 60, no. 2, p. 154-159, Jun. 2014.

[4] S. I. Park, et al,"A Newly Designed Quarter-Rate QC-LDPC Code for the Cloud Transmission System". IEEE Transaction on Broadcasting, vol. 59, no. 1, Mar. 2013.

[5] Y. Wu, et al,"Cloud Transmission: A New Spectrum-Reuse Friendly Digital Terrestrial Broadcasting Transmission System" IEEE Transaction on Broadcasting, vol. 58, no. 2, Set. 2012.

[6] ATSC, "Summaries of Responses to ATSC 3.0 Physical Layer Call for Proposals," Out. 2013.

[7] ATSC, "Call for Proposals For ATSC 3.0 PHYSICAL LAYER - A Terrestrial Broadcast Standard," Mar. 2013.

[8] ATSC, "ATSC 3.0 Going international with 2015 NAB Show demonstrations". [Online]. Disponível em: http://atsc.org/newsletter/atsc-3-0-going-international-with-2015-nabshow-demonstrations/?zoom_highlight=ldm.

[9] S. I. Park, et al. "Hardware Implementation and Complexity Analysis ofLayered Division Multiplexing (LDM) System for ATSC 3.0". em NAB Broadcast Engineering Conference Proceedings. Las Vegas, Nevada. 2015, p77-81.

[10] S. Takeuchi, et al. "Super Hi-Vision Multimedia Broadcast Systems Based on "Hybridcast"'. em NAB Broadcast Engineering Conference Proceedings. Las Vegas, Nevada. 2015, p181-185.

[11] NHK STRL, “Annual Report 2013,” NHK STR. Toquio Ago. 2014.

[12] S. Saito, et al, "Technology for next-generation digital terrestrial broadcasting - Field experiments of $8 \mathrm{~K}$ Super Hi-Vision transmission using dual-polarized MIMO and ultra-multilevel OFDM" IEEE International Symposium on Broadband Multimedia Systems and Broadcasting, vol. 60, no. 2, p. 1-6 Jun. 2014.

[13] ETSI, TS 102831 v1.2.1. Digital Video Broadcasting (DVB) Implementation guidelines for a second generation digital terrestrial television broadcasting system (DVB-T2). Ago. 2012.

[14] J. Montalban, et al "Cloud Transmission: System Simulation and Performance Analysis". IEEE International Symposium on Broadband Multimedia Systems and Broadcasting. p 1-5, Jun 2013.

[15] L. Yaqi, et al. "On the performance of ldpc-rs product codes for mobile dtv". IEEE International Symposium on Broadband Multimedia Systems and Broadcasting. p 1-4, Jun. 2012.

[16] U. Gil et al. "Cloud transmission system performance for mobile urban scenarios in the field". IEEE International Symposium on Broadband Multimedia Systems and Broadcasting, p. 1 - 5, Jun. 2014.

[17] T. Shitomi, et al. "Study on circularly arranged non-uniform constellations in dual-polarized MIMO-OFDM transmission" IEEE Broadband Multimedia Systems and Broadcasting (BMSB). p 1-4, Jun 2014.

[18] D. He. et al. "Improvements to apsk constellation with gray mapping" IEEE International Symposium on Broadband Multimedia Systems and Broadcasting. p 1-4, Jun. 2014.

[19] J. Zoellner, N. Loghin. "Optimization of High-order Non-uniform QAM Constellations". IEEE International Symposium on Broadband Multimedia Systems and Broadcasting. p 1-4, Jun. 2013.

[20] B. Mouhouche, D. Ansorregui, A. Mourad.” High Order Non-Uniform Constellations for broadcasting UHDTV". IEEE Wireless Communications and Networking Conference. p 600 - 605 Abr. 2014

[21] L. Polak, T. Kratochvil. "Comparison of the Non-rotated and Rotated Constellations Used in DVB-T2 Standard". $22^{\text {nd }}$ International Conference Radioelektronika, p. 1- 4, Abr. 2012.

[22] M. Breiling, J. Zöllner, J. Robert. "When do rotated constellations provides gain?". IEEE International Symposium on Broadband Multimedia Systems and Broadcasting, p. 1 - 6, Jun. 2014.

\footnotetext{
Cite this article:

F. Leone, L., Akamine, C.; 2015. Técnicas de codificação de canal e modulação para a próxima geração de televisão digital. SET EXPO PROCEEDINGS. ISSN Print: 2447-0481.ISSN Online: 2447-049X. v.1. doi: 10.18580/setep.2015.1.8 Web-link: http://dx.doi.org/10.18580/

setep.2015.1.8
} 